\title{
Susceptibility Pattern of Bacille Calmette-Guerin Strains Against Pyrazinamide and Other Major Anti-Mycobacterial Drugs
}

\author{
Seyed Alireza Fahimzad ${ }^{1}$; Mahmood Ghasemi ${ }^{2}$; Farideh Shiva ${ }^{1}$; Keyghobad Ghadiri ${ }^{2}$; \\ Masoumeh Navidinia ${ }^{3}$; Abdollah Karimi ${ }^{1,}$ \\ ${ }_{1}^{1}$ Pediatric Infections Research Center, Mofid Children's Hospital, Shahid Beheshti University of Medical Sciences, Tehran, IR Iran \\ ${ }^{2}$ Nosocomial Infection Research Center, Kermanshah University of Medical Sciences, Kermanshah, IR Iran \\ ${ }^{3}$ Department of Laboratory Sciences, Paramedical Sciences Faculty, Shahid Beheshti University of Medical Sciences, Tehran, IR Iran \\ ${ }^{*}$ Corresponding author: Abdollah Karimi, Pediatric Infections Research Center, Mofid Children's Hospital, Shahid Beheshti University of Medical Sciences, Tehran, IR Iran. Tel/Fax: \\ +98-2122226941, E-mail: info@pedirc.org
}

Received: January 24, 2014; Revised: March 12, 2014; Accepted: June 28, 2014

\begin{abstract}
Background: BCG (Bacille Calmette-Guerin) vaccine is an attenuated live vaccine administered to prevent Tuberculosis. Disseminated infection due to BCG is a rare life threatening complication of this vaccine, especially in immunocompromised patients.

Objectives: The current study evaluated the sensitivity pattern of the BCG strain supplied by the Institute de Pasteur, Iran against antimycobacterial drugs used to treat disseminated infection caused by the vaccine.

Patients and Materials: Bacille Calmette-Guerin strain ATCC 1173 P was purchased from the Pasteur Institute in Tehran and three samples of Mycobacterium bovis isolated from subaxillary adenitis of three patients were tested for susceptibility to major anti-mycobacterial drugs by MODS (microscopic observation drug sensitivity) method.

Results: All samples of BCG strain were resistant to most of the anti-mycobacterial drugs used separately except for concentrations of isoniazid $1 \mu \mathrm{g} / \mathrm{mL}$, ethambutol $3 \mu \mathrm{g} / \mathrm{mL}$, ciprofloxacin $4 \mu \mathrm{g} / \mathrm{mL}$ and clarithromycin $2 \mu \mathrm{g} / \mathrm{mL}$. Addition of pyrazinamide (PZA) $25 \mu \mathrm{g} / \mathrm{mL}$, the susceptibility pattern did not change to any drug, but increasing PZA concentration to $50 \mu \mathrm{g} / \mathrm{ml}$ and combining it with ethionamide $2 \mu \mathrm{g} / \mathrm{mL}$, isoniazid $0.2 \mu \mathrm{g} / \mathrm{mL}$, streptomycin $2 \mu \mathrm{g} / \mathrm{mL}$, rifampin $0.5 \mu \mathrm{g} / \mathrm{mL}$ or ciprofloxacin $0.2 \mu \mathrm{g} / \mathrm{mL}$, made the Mycobacterium strains susceptible.

Conclusions: The current study findings revealed that Mycobacterium bovis resistance to pyrazinamide is resolved by increasing the concentration of the drug and combining PZA with other anti-mycobacterial agents.
\end{abstract}

Keywords: Mycobacterium bovis; Pyrazinamide; Resistance

\section{Background}

It is estimated that one-third of the world population are infected with Tuberculosis (TB); though a curable disease, it remains as a global problem with the increasing number of cases infected with drug-resistant mycobacteria spp. Most of the cases are observed in developing countries. Approximately 1.7 million people die every year due to TB and more than 400000 cases of multidrug-resistant TB emerge every year; the disease cases were reported from almost all regions of the world in 2006 (13). To prevent severe forms of Tuberculosis, BCG, (Bacille Calmette-Guerin) vaccine has been administrated worldwide including Iran. The BCG vaccine was developed from 1908 to 1921 by Albert Calmette and Camille Guerin in France by culturing Mycobacterium bovis on bile containing medium. In 1921 it was used in human in France. Bacille Calmette-Guerin immunization has been used in Iran since 1948 for mass vaccination (4). Evidences from animals and humans showed significant differences in the immune responses induced by different BCG vaccine strains (5). It is a safe vaccine made from attenuated live bacteria, but sporadic cases of local or systemic diseases have been reported in vaccine recipients. The systemic infection of BCG may be life threatening especially in immunocompromised patients. When the systemic infections are diagnosed, anti-TB therapy is started empirically before obtaining the susceptibility results (6). In order to ensure the optimal outcome of the treatment, it is important to determine the susceptibility of the BCG Mycobacterium to the prescribed antibiotic regimen.

\section{Objectives}

The current study aimed to measure the minimum inhibitory concentration (MIC) of pyrazinamide, (PZA), and some other anti-mycobacterial drugs against the strain of BCG supplied by the Institute de Pasteur in Iran to routinely vaccinate the newborns against TB.

\section{Patients and Methods}

Bacille Calmette-Guerin strain ATCC $1173 \mathrm{P}$ and three samples of Mycobacterium bovis isolated from subaxillary adenitis of three patients were used in this study. 
Fahimzad SA et al.

The tested antibiotics in the current study included PZA, isoniazid, rifampin, ethambutol, streptomycin, ciprofloxacin, clarithromycin and ethionamide; all antibiotics were provided by Sigma Company (cat No: 0123K568711). Susceptibility testing was performed by MODS (microscopic observation drug sensitivity) method. Direct drug-susceptibility testing was performed by the MODS assay. The used drug concentrations were as follows: PZA 25 and $50 \mu \mathrm{g} / \mathrm{mL}$, isoniazid 0.2 and $1 \mu \mathrm{g} /$ $\mathrm{mL}$, rifampin 0.5 and $1 \mu \mathrm{g} / \mathrm{mL}$, ethambutol 2 and $3 \mu \mathrm{g} /$ $\mathrm{mL}$, streptomycin $2 \mu \mathrm{g} / \mathrm{mL}$, ciprofloxacin 0.2 and $4 \mu \mathrm{g} /$ $\mathrm{mL}$, clarithromycin $2 \mu \mathrm{g} / \mathrm{mL}$, and ethionamide 2 and 3 $\mu \mathrm{g} / \mathrm{mL}$. The growth detection procedure in MODS was as follows: $50 \mu \mathrm{L}$ of 0.5 McFarland suspension of BCG strain was inoculated into $7.2 \mathrm{~mL}$ of Middle brook 7H9 broth (Difco) containing $5.9 \mathrm{~g}$ of Middlebrook 7H9 broth base per liter, 0.31\% glycerol, $1.25 \mathrm{~g}$ of Bacto Casitone (Difco) per liter, 10\% OADC, and $160 \mu \mathrm{L}$ of PANTA antimicrobial Supplement Stock $(20 \mu \mathrm{L} / \mathrm{mL})$. For each sample, aliquots of $1.2 \mathrm{~mL}$ (each) were distributed to 6 wells of a sterile 24-well plate (Falcon, Franklin Lakes, N.J.). To improve microscopic visualization of the bacteria, a 24-well plate was used rather than a 96-well plate. When three specimens were run, the fourth set of 6 wells was filled with broth only to control cross-contamination. Each run also included a susceptible control of Mycobacterium bovis strain H37Rv in which a 1.50 dilution of 0.5 McFarland standard equivalent in broth was added to one well. The growth was examined daily between the sixth and eighth days. Plates were sealed with scotch polyethylene tape (Fisher, Springfield, N.J.) and incubated at $37^{\circ} \mathrm{C}$ for up to 40 days. Aliquots were examined daily under an inverted light microscope at 40X.(Figure 1) Each well was examined for approximately 30 to 45 seconds (7).

\section{Results}

Bacilli were uniformly sensitive to isoniazid $1 \mu \mathrm{g} / \mathrm{mL}$, ethambutol $3 \mu \mathrm{g} / \mathrm{mL}$, ciprofloxacin $4 \mu \mathrm{g} / \mathrm{mL}$, and clarithromycin $2 \mu \mathrm{g} / \mathrm{mL}$ (Table 1). All samples of BCG strain Mycobacterium were resistant to PZA 25 and $50 \mu \mathrm{g} / \mathrm{mL}$, isoniazid $0.2 \mu \mathrm{g} / \mathrm{mL}$, ethambutol $2 \mu \mathrm{g} / \mathrm{mL}$, ethionamide
$3 \mu \mathrm{g} / \mathrm{mL}$, rifampin $1 \mu \mathrm{g} / \mathrm{mL}$, ciprofloxacin $0.2 \mu \mathrm{g} / \mathrm{mL}$, and streptomycin $2 \mu \mathrm{g} / \mathrm{mL}$. The organism showed resistance against a combination of any of the above drugs with PZA $25 \mu \mathrm{g} / \mathrm{mL}$, but on increasing the concentration of PZA to $50 \mu \mathrm{g} / \mathrm{mL}$ and combining it with ethionamide $2 \mu \mathrm{g} / \mathrm{mL}$, isoniazid $0.2 \mu \mathrm{g} / \mathrm{mL}$, streptomycin $2 \mu \mathrm{g} / \mathrm{mL}$, rifampin $0.5 \mu \mathrm{g} / \mathrm{mL}$ or ciprofloxacin $0.2 \mu \mathrm{g} / \mathrm{mL}$, the $M y$ cobacterium spp. failed to grow and was observed to be susceptible (Table 1). Susceptibility patterns with other various combinations of drugs detected in this study are shown in Table 2.

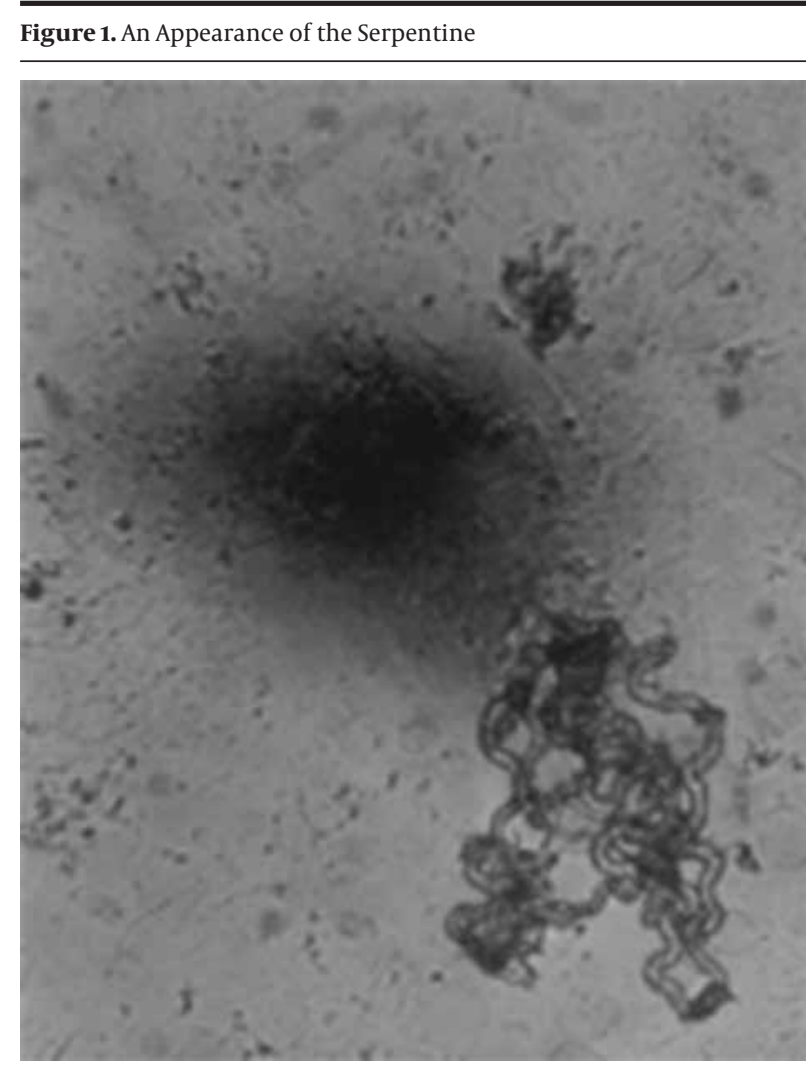

Ropy aspect of the BCG micro-colonies at 100X after 25 days of broth culturing.

Table 1. Sensitivity Pattern of Mycobacterium bovis Singly or in Combination With Pyrazinamide $(25 \text { or } 50 \mu \mathrm{g} / \mathrm{mL})^{\mathrm{a}}$

\begin{tabular}{|c|c|c|c|c|c|c|c|c|c|c|c|c|c|}
\hline 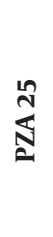 & in & 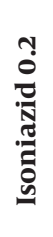 & 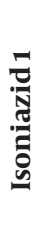 & 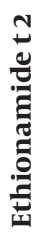 & 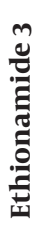 & 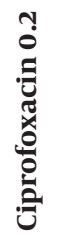 & 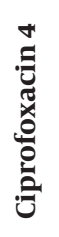 & 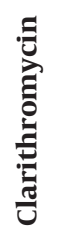 & 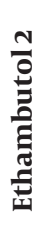 & 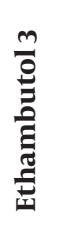 & 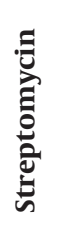 & 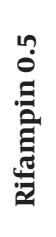 & 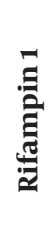 \\
\hline $\mathrm{R}$ & $\mathrm{R}$ & $\mathrm{R}$ & $S$ & $\mathrm{R}$ & $\mathrm{R}$ & $\mathrm{R}$ & $S$ & $S$ & $\mathrm{R}$ & $S$ & $\mathrm{R}$ & $\mathrm{R}$ & $\mathrm{R}$ \\
\hline- & - & $\mathrm{R}$ & $S$ & $\mathrm{R}$ & $\mathrm{R}$ & $\mathrm{R}$ & $S$ & $S$ & $\mathrm{R}$ & $S$ & $\mathrm{R}$ & $\mathrm{R}$ & $\mathrm{R}$ \\
\hline- & - & $\mathrm{R}$ & $S$ & $S$ & $S$ & $\mathrm{R}$ & $S$ & $S$ & $\mathrm{R}$ & $S$ & $\mathrm{R}$ & $\mathrm{R}$ & $\mathrm{R}$ \\
\hline
\end{tabular}

\footnotetext{
a Abbreviations: PZA, pyrazinamide; R, Resistance; $S$, sensitive.
} 
Fahimzad SA et al.

Table 2. Sensitivity Pattern of Mycobacterium bovis in Combination of Two Antibiotics With Pyrazinamide (25 or $50 \mu \mathrm{g} / \mathrm{mL})$

\begin{tabular}{|c|c|c|c|c|c|c|c|c|c|c|c|c|}
\hline 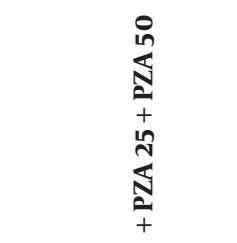 & 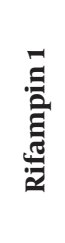 & 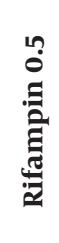 & 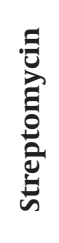 & 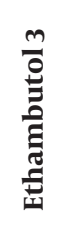 & 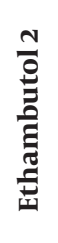 & 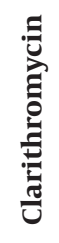 & 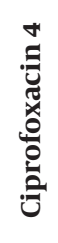 & 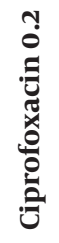 & 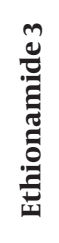 & 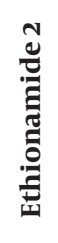 & 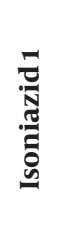 & 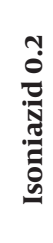 \\
\hline Isoniazid 0.2 & $\mathrm{RR}$ & $\mathrm{RR}$ & RS & SS & $\mathrm{RR}$ & - & - & - & - & - & - & - \\
\hline Isoniazid 1 & RS & RS & SS & SS & $\mathrm{RR}$ & - & - & - & - & - & - & - \\
\hline Ethionamide 2 & - & - & - & - & - & SS & RS & RS & - & - & - & - \\
\hline Ethionamide 3 & - & - & - & - & - & SS & RS & RS & - & - & - & - \\
\hline Ciprofloxacio.2 & - & - & - & - & - & $\mathrm{RR}$ & - & - & RS & RS & - & - \\
\hline Ciprofloxacin 4 & - & - & - & - & - & SS & - & - & RS & RS & - & - \\
\hline Clarithromycin & - & - & - & - & - & - & SS & $\mathrm{RR}$ & SS & SS & - & - \\
\hline ethambutol 2 & $\mathrm{RR}$ & $\mathrm{RR}$ & $\mathrm{RR}$ & - & - & - & - & - & - & - & $\mathrm{RR}$ & $\mathrm{RR}$ \\
\hline Ethambutol 3 & SS & SS & SS & - & - & - & - & - & - & - & SS & SS \\
\hline Streptomycin & $\mathrm{RR}$ & $\mathrm{RR}$ & - & SS & $\mathrm{RR}$ & - & - & - & - & - & SS & $\mathrm{RS}$ \\
\hline Rifampin 0.5 & - & - & $\mathrm{RR}$ & SS & $\mathrm{RR}$ & - & - & - & - & - & RS & $\mathrm{RR}$ \\
\hline Rifampin 1 & - & - & $\mathrm{RR}$ & SS & $\mathrm{RR}$ & - & - & - & - & - & RS & $\mathrm{RR}$ \\
\hline
\end{tabular}

\section{Discussion}

Tuberculosis (TB) infects $20 \%$ to $45 \%$ of the world population. In terms of morbidity and mortality, it is one of the most important infections in the world (8). Bacille Calmette-Guerin is one of the first live attenuated vaccines used in humans. About 1\% of the children receiving this vaccine develop local adverse reactions which are not generally serious and include subcutaneous abscesses and lymphadenopathy (9). In about one per one million doses, vaccine recipients may develop osteitis that might occur several years after BCG vaccination. Disseminated fatal disease rarely occurs in children; its rate varies between 0.1 and 1 per million doses and it is primarily observed in immunocompromised children (10-12). Disseminated infection and osteitis due to BCG require systemic treatment with anti-mycobacterial drugs. Although PZA, a synthetic Pyrazide with bactericidal anti-mycobacterial activity, is used to treat Mycobacterium tuberculosis, Mycobacterium bovis is known to be resistant to this drug (13). The latest international recommendations, from the World Health Organization (WHO) and the European Centre for Disease Prevention and Control (ECDC), do not include this drug in the anti-tuberculosis drugs group to be routinely tested (14). On the other hand, due to the potential for major errors during PZA susceptibility testing with the M960 assay, laboratories should consider retesting all PZA-resistant isolates to provide accurate and reliable susceptibility results (15). According to the study from 2001 to 2004 in New York city, 49\% of the 35 positive Mycobacterium bovis isolates showed resistance against PZA alone, 40\% against a combination of PZA and streptomycin, and $6 \%$ against PZA, isoniazid and streptomycin
(16). In the current study, when PZA with high concentrations was added to the culture plates $(50 \mu \mathrm{g} / \mathrm{mL})$ in combination with other anti-mycobacterial agents, Mycobacterium bovis failed to grow in spite of the initial resistance to PZA alone. Shishido et al. found that Mycobacterium bovis BCG Tokyo strain was susceptible to all major antituberculosis drugs, (isoniazid, rifampin, streptomycin and ethambutol) but resistant to PZA (17). Similar results were observed by Durek et al. (18). In contrast to these observations, in the current study Mycobacterium bovis Pasteur strain was resistant to major anti-tuberculosis drugs such as rifampin and streptomycin in addition to PZA and other new drugs like moxifloxacin. The major limitation of the current research was the in vitro experiments conducted on a few strains of Mycobacterium bovis, therefore complementary researches are needed to apply in vivo cases. Since disseminated BCG needs multi-drug therapy and drug resistant strains are increasing in the recent years, there is a need to look for new options in the therapy. Findings of the current study illustrated that although the Mycobacterium bovis strain was resistant to PZA in all cases, on increasing PZA concentration from 25 to $50 \mu \mathrm{g} / \mathrm{mL}$ and adding other anti-tuberculous drugs to the suspension, the microorganism revealed susceptibility to the combined regimen. If other in vivo studies show that high level of PZA in blood has no toxicity, there will be new options in the disseminated BCG infections management.

\section{Acknowledgements}

Authors are grateful to the staff of the Pediatric Infec- 
tions Research Center, Tehran, Iran for making this research possible.

\section{Authors' Contributions}

Study concept and design: Dr. Fahimzad and Dr. Karimi; acquisition of data: Dr. Ghasemi; analysis and interpretation of data: Dr. Shiva and Dr. Navidinia; drafting of the manuscript: Dr. Fahimzad, Dr. Ghadiri; critical revision of the manuscript for important intellectual content: Dr. Ghadiri, Dr. Shiva; study supervision: Dr. Fahimzad and Dr. Karimi.

\section{Funding/Support}

The current study received funding supports from Pediatric Infection Research Center (PIRC).

\section{References}

1. Netto EM, Dye C, Raviglione MC. Progress in global tuberculosis control 1995-1996, with emphasis on 22 high-incidence countries. Global Monitoring and Surveillance Project. Int J Tuberc Lung Dis. 1999;3(4):310-20.

2. Centers for Disease Control and Prevention. Reported tuberculosis in the United States, 2006. In: Department of Health and Human, editor. Atlanta: CDC; 2007.

3. Zignol M, Hosseini MS, Wright A, Weezenbeek CL, Nunn P, Watt CJ, et al. Global incidence of multidrug-resistant tuberculosis. J Infect Dis. 2006;194(4):479-85.

4. Ritz N, Hanekom WA, Robins-Browne R, Britton WJ, Curtis N. Influence of BCG vaccine strain on the immune response and protection against tuberculosis. FEMS Microbiol Rev. 2008; 32(5):821-41.

5. The Pasteur Institute of Iran seen by Marcel Baltazard. Iran; 2004. Booklet published by the Cooperation and Cultural Action of the Embassy of France in IR Iran.

6. Lotte A, Wasz-Hockert O, Poisson N, Dumitrescu N, Verron M, Cou- vet E. BCG complications. Estimates of the risks among vaccinated subjects and statistical analysis of their main characteristics. Adv Tuberc Res. 1984;21:107-93.

7. Caviedes L, Lee TS, Gilman RH, Sheen P, Spellman E, Lee EH, et al. Rapid, efficient detection and drug susceptibility testing of Mycobacterium tuberculosis in sputum by microscopic observation of broth cultures. The Tuberculosis Working Group in Peru. JClin Microbiol. 2000;38(3):1203-8.

8. Jeffrey RS. Tuberculosis. In: Feigin RD, Cherry J, Demler-Harrison GJ, kaplan SL editors. Textbook of Pediatric Infectious Diseases. 6th ed; 2009. pp. 1426-69.

9. Nazir Z, Qazi SH. Bacillus Calmette-Guerin (BCG) lymphadenitischanging trends and management. J Ayub Med Coll Abbottabad. 2005;17(4):16-8.

10. Turnbull FM, McIntyre PB, Achat HM, Wang H, Stapledon R, Gold $M$, et al. National study of adverse reactions after vaccination with bacille Calmette-Guerin. Clin Infect Dis. 2002;34(4):447-53.

11. Deeks SL, Clark M, Scheifele DW, Law BJ, Dawar M, Ahmadipour $\mathrm{N}$, et al. Serious adverse events associated with bacille CalmetteGuerin vaccine in Canada. Pediatr Infect Dis J. 2005;24(6):538-41.

12. Fine P, E. M, Carneiro IAM, Milstien J, B, Clements CJ. Issues relating to the use of BCG in immunization programmes. World Health Organization. 1999;45.

13. de Jong BC, Onipede A, Pym AS, Gagneux S, Aga RS, DeRiemer K, et al. Does resistance to pyrazinamide accurately indicate the presence of Mycobacterium bovis? J Clin Microbiol. 2005;43(7):3530-2.

14. Cunha J. Pyrazinamide susceptibility testing in all isolates of the Mycobacterium tuberculosis complex - a critical analysis. Rev Port Pneumol. 2012;18(4):188-9.

15. Piersimoni C, Mustazzolu A, Giannoni F, Bornigia S, Gherardi G, Fattorini L. Prevention of false resistance results obtained in testing the susceptibility of Mycobacterium tuberculosis to pyrazinamide with the Bactec MGIT 960 system using a reduced inoculum. JClin Microbiol. 2013;51(1):291-4.

16. Centers for Disease Control and Prevention (CDC). Human tuberculosis caused by Mycobacterium bovis--New York City, 20012004. MMWR Morb Mortal Wkly Rep. 2005;54(24):605-8.

17. Shishido Y, Mitarai S, Otomo K, Seki M, Sato A, Yano I, et al. Antituberculosis drug susceptibility testing of Mycobacterium bovis BCG Tokyo strain. Int JTuberc Lung Dis. 2007;11(12):1334-8.

18. Durek C, Rusch-Gerdes S, Jocham D, Bohle A. Sensitivity of BCG to modern antibiotics. Eur Urol. 2000;37 Suppl 1:21-5. 